\title{
ITGA7 functions as a tumor suppressor and regulates migration and invasion in breast cancer
}

This article was published in the following Dove Press journal:

Cancer Management and Research

\section{Adheesh Bhandari* \\ Erjie Xia* \\ Yuying Zhou \\ Yaoyao Guan \\ Jingjing Xiang \\ Lingguo Kong \\ Yinghao Wang \\ Fan Yang \\ Ouchen Wang \\ Xiaohua Zhang}

Department of Thyroid and Breast Surgery, The First Affiliated Hospital of Wenzhou Medical University,

Wenzhou, Zhejiang, People's Republic of China

*These authors contributed equally to this work
Correspondence: Ouchen Wang;

Xiaohua Zhang

Department of Thyroid and Breast Surgery, The First Affiliated Hospital of Wenzhou Medical University, Nanbai Xiang, Wenzhou 325000, Zhejiang,

People's Republic of China

Tel +86 I39 $57706099 ;+86$ I36 0067

1188

Email woc863@hotmail.com; oncology_ zhang@outlook.com
Background: Breast cancer is the most common malignancy in women and the underlying mechanism of breast cancer cell metastasis is still far from uncover. Integrin subunit alpha 7 (ITGA7) is a functioning protein. It has been detected in many malignancies. But the function of ITGA7 in breast cancer is not clear. Our aim is to explore ITGA7 expression and its role in breast cancer. Methods: Real-time PCR was performed to determine ITGA7 expression in BC tissues and normal adjacent tissues. The specific functions of ITGA7 in breast cancer cell lines (MDA-MB-231 and BT-549) transfected with small interfering RNA were determined through migration, invasion assays. Western blot assays were performed to determine the expression of c-met and vimentin. Results: ITGA7 was down-regulated in breast cancer tissues compared to the adjacent normal tissues (T:N $=7.68 \pm 27.38: 41.01 \pm 31.47, P<0.001)$ and this observation was consistent with the TCGA cohort (T:N $=4.51 \pm 0.45: 5.40 \pm 0.61, P<0.0001)$. In vitro experiments showed that knocking down ITGA7 significantly inhibited the migration and invasion of the breast cancer cell lines (MDA-MB-231 and BT-549). Meanwhile, knockdown of ITGA7 promoted c-met and vimentin expression, which may induce invasion and migration.

Conclusion: ITGA7 plays an important tumorigenic function and acts as a suppress gene in breast cancer. Our findings indicate that $I T G A 7$ was the gene associated with breast cancer.

Keywords: breast cancer, ITGA7, migration, invasion

\section{Introduction}

Breast cancer is the most common malignancy in women. As the reason for cancerrelated death, breast cancer ranks second after lung cancer. ${ }^{1,2}$ Many studies have proven that invasion of cancer cells was the major reason for cancer-related death. ${ }^{3,4}$ With acknowledging development of medical technology, doctors can treat breast cancer patients by surgery, chemotherapy, endocrine therapy, or targeted therapies. ${ }^{5}$ But the treatment is not always satisfactory. So, it is still important to explore the mechanism of breast cancer and find new biomarkers at an early stage.

C-met is a key regulator of cancer progression, and it has been reported that upregulated c-met is related with poor survival rates and malignant activities of breast cancer. ${ }^{6,7}$ During cancer progression, cancer cells can experience a feature change from an epithelial to a mesenchymal phenotype, which is called epithelial-mesenchymal transition (EMT).${ }^{8,9}$ Hung et $\mathrm{al}^{10}$ found that osthole suppresses HGF-induced EMT via repression of the c-Met/Akt/mTOR pathway in human breast cancer cells.

Integrin subunit alpha 7 (ITGA7) belongs to the integrin alpha chain family, and the coding gene is located on chromosome $12 \mathrm{q} 13.2 .{ }^{11}$ It has been found that ITGA7 is expressed in many cancers including malignant melanoma, prostate and liver submit your manuscript

Dovepress

f

in 
carcinomas, and glioblastoma. ${ }^{12,13}$ Low levels of ITGA7 mediate cell adhesion migration on specific laminin isoforms and influence tumors growth and motility. ${ }^{14}$ Ziober et al ${ }^{12}$ found that upregulation of ITGA7 could reduce melanoma cell tumor growth, motility, and metastasis. ${ }^{12}$ Ren et al ${ }^{13}$ found that downregulation of ITGA7 expression increased the rate of migration in lung cancer cells. However, the relationship between ITGA7 and breast cancer is not exactly clear.

In order to clarify the expression and effect of ITGA7 in breast cancer, our study examined ITGA7 expression in breast cancer tissues and paired adjacent nontumor tissues by using real-time quantitative PCR (RT-qPCR), and we verified our clinical specimen data by using The Cancer Genome Atlas (TCGA) database. Besides, we characterized the function of $I T G A 7$ in breast cancer cell lines. We found that downregulated $I T G A 7$ could promote breast cancer cell migration and invasion.

\section{Materials and methods}

\section{Patients and breast tissue samples}

In this present study, we obtained 36 breast cancer tissues and paired adjacent nontumor tissues from the Department of Thyroid and Breast Surgery, The First Affiliated Hospital of Wenzhou Medical University, Wenzhou, Zhejiang, People's Republic of China. All patient-derived specimens and information were collected and recorded based on the protocols provided by the Ethics Committee of The First Affiliated Hospital of Wenzhou Medical University. These 36 fresh tissues were snap-frozen in liquid nitrogen immediately and stored at $-80^{\circ} \mathrm{C}$. Breast cancer mRNA expression data were downloaded from the TCGA data portal (https://tcga-data.nci. nih.gov/tcga/). Gene expression data were available for 1,100 breast cancer samples compared to 113 normal samples.

\section{Ethical approval}

Ethical approval for this study was obtained from the Ethics Committee of the First Affiliated Hospital of Wenzhou Medical University.

\section{Cell cultures and growth conditions} MDA-MB-231, BT-549, SK-BR-3, MDA-MB-468, MCF7 , and MCF-10A cells were used in this study. These cells were obtained from Shanghai Cell Biology, Institute of the Chinese Academy of Sciences (Shanghai, People's Republic of China). MDA-MB-231, MCF-7, and SK-BR-3 were cultured in Dulbecco's Modified Eagle's Medium (DMEM) (Gibco, Grand Island, NY, USA) supplemented with 10\% fetal bovine serum (FBS) (Gibco). BT-549 were cultured in Roswell Park Memorial Institute-1640 medium (Gibco) supplemented with
10\% FBS (Gibco). MDA-MB-468 cells were cultured in L-15 medium (Gibco) supplemented with 10\% FBS (Gibco). MCF10A cells were cultured in DMEM-F12 (Gibco) supplemented with $100 \mathrm{U} / \mathrm{mL}$ of penicillin, $100 \mu \mathrm{g} / \mathrm{mL}$ of streptomycin, 2 $\mathrm{mM}$ of L-glutamine, $20 \mathrm{ng} / \mathrm{mL}$ of epidermal growth factor, and 10\% FBS (Gibco) MDA-MB-468 cells were incubated in a standard cell culture incubator (Thermo, Waltham, MA, USA) at $37^{\circ} \mathrm{C}$ without $\mathrm{CO}_{2}$. The others were incubated in a standard cell culture incubator (Thermo) at $37^{\circ} \mathrm{C}$ with $5 \% \mathrm{CO}_{2}$.

\section{Cell transfection}

MDA-MB-231 and BT-549 were transfected using Lipofectamine RNAiMAX transfection reagent (Invitrogen, Carlsbad, CA, USA) by following the manufacturer's protocol. About 100,000 cells were plated 1 day before transfection. ITGA7 was silenced by transfecting $10 \mathrm{nM}$ siRNA for $48 \mathrm{~h}$. The siRNA sequences used in the study were the ITGA7 siRNAs that targeted the following sequences: ITGA 7 siRNA-1, forward 5'-GCAUCAAGAGCUUCGGCUATT-3' and reverse 5'-UAGCCGAAGCUCUUGAUGCTT-3'; ITGA 7 siRNA-2, forward $5^{\prime}$-GCUGCCCACUCUACAGCUUTT- $3^{\prime}$ and reverse 5'-AAGCUGUAGAGUGGGCAGCTT-3'; ITGA7 siRNA-3, forward 5'-GGUCAUCCUCCUGGCUGUATT-3' and reverse 5'-UACAGCCAGGAGGAUGACCTT-3'. Both siRNAs were provided by Genepharma (Shanghai, People's Republic of China) company.

\section{RNA extraction and RT-qPCR}

Total RNA was lysed using TRIzol reagent according to the manufacturer's instructions (Invitrogen). The purity of the isolated RNA was measured at $260 / 280 \mathrm{~nm}$ by spectrophotometry (Thermo). After measurement, RNA samples were stored at $-80^{\circ} \mathrm{C}$. Real-time reactions were run and analyzed by using a real-time PCR system (Applied Biosystems 7500, ThermoFisher Scientific). The relative expression of mRNA was calculated using the comparative cycle threshold (CT) $\left(2^{-\Delta \Delta \mathrm{CT}}\right)$ method with GAPDH as the endogenous control to normalize the data. The sequences of the primers used were as follows:

ITGA7 forward: 5'-GCTGTGAAGTCCCTGGAAGT GATT- $3^{\prime}$ and reverse: $5^{\prime}$-GCATCTCGGAGCATCAAGTTC TT-3'; GADPH forward: 5'-GTCTCCTCTGACTTCA ACAGCG-3' and reverse: 5'-ACCACCCTGTTGCTGTA GCCAA-3'.

\section{Invasion and migration assay}

For cell Transwell assays, cells were trypsinized with trypsin and collected in the medium containing 10\% FBS. Invasion of cells was measured in Matrigel (BD, Franklin Lakes, NJ, 
USA)-coated Transwell inserts $(6.5 \mathrm{~mm}$, Costar, Manassas, VA, USA) containing polycarbonate filters with $8 \mu \mathrm{m}$ pores. The inserts were coated with $50 \mu \mathrm{L}$ of $1 \mathrm{mg} / \mathrm{mL}$ Matrigel matrix according to the manufacturer's recommendations. A total of 40,000 cells $(-250 \mu \mathrm{L})$ were transferred into the upper chamber. About $700 \mu \mathrm{L}$ medium containing $20 \% \mathrm{FBS}$ was filled in the lower chamber. Then the plate was placed into the incubator. After $24 \mathrm{~h}$, the membrane was fixed with $4 \%$ paraformaldehyde and stained with $0.4 \%$ crystal violet solution for $15 \mathrm{~min}$. Motility assays were similar to invasion assay except that the Transwell insert was not coated with Matrigel. Cell migration and invasion ability were assessed by counting the cells that had migrated and invaded through the membrane. Five random fields of view were selected and images captured under a microscope at a magnification of $20 \times$.

\section{Western blot analysis}

Whole cell lysates were separated by $10 \%$ sodium dodecyl sulfate-polyacrylamide gels electrophoresis (BioRad, Berkeley, CA, USA) and transferred to a PVDF membrane (Millipore, Billerica, MA, USA). The membranes were blocked with $5 \%$ nonfat milk for $2 \mathrm{~h}$ at room temperature. According to the manufacturer's protocol, the membranes were probed with polyclonal antibody overnight at $4^{\circ} \mathrm{C}$. The membranes were then incubated with the anti-mouse IgG or anti-rabbit IgG as secondary antibody (Abcam, Cambridge, MA, USA) for $1 \mathrm{~h}$ at room temperature. The primary antibodies used were as follows: Vimentin (Abcam, ab92547), ITGA7 (Abcam, ab203254), and human GAPDH (Sigma, St. Louis, MO, USA).

\section{Statistical analysis}

All statistical analyses were performed using SPSS 23.0 software (IBM Corporation, Armonk, NY, USA). Data are presented as mean \pm standard error. The differences were considered to be statistically significant at $P<0.05$. Student's $t$-test (2-tailed) was performed to analyze differences between groups.

\section{Result \\ ITGA7 was downregulated in breast cancer tissues}

In order to investigate the function of ITGA7 in breast cancer tumorigenesis, the expression levels of ITGA7 were investigated in 36 breast cancer tissues and paired adjacent nontumor tissues by using RT-qPCR. We found that ITGA7 expression was significantly lower in breast cancer tissues, compared to the adjacent normal tissues $(\mathrm{T}: \mathrm{N}=7.68 \pm 27.38: 41.01 \pm 31.47$, $P<0.001$ ) (Figure 1A). TCGA also showed that ITGA7 was downregulated in breast cancer compared to the adjacent normal tissues $(\mathrm{T}: \mathrm{N}=4.51 \pm 0.45: 5.40 \pm 0.61, P<0.001)$ (Figure 1B). In a word, these results implied that $I T G A 7$ might function as a tumor suppressor in breast cancer.

\section{The relationship between ITGA7 expression and clinical features}

To understand the relation between ITGA7 and breast cancer, we investigated the relationship of ITGA7 with clinicopathological features. In the TCGA cohort, we divided the patients into low-expression group and high-expression
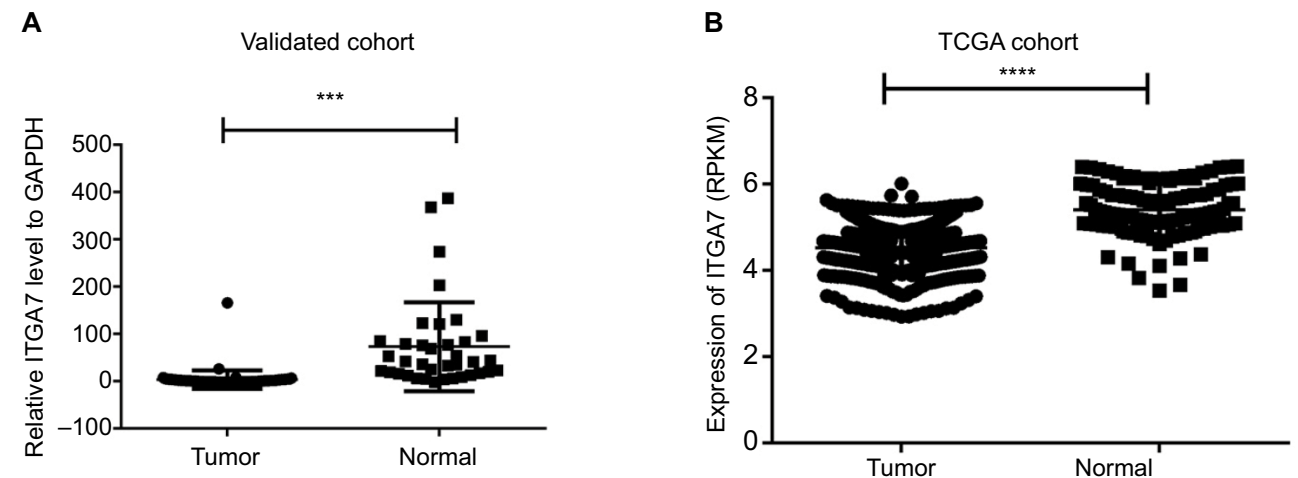

Figure I ITGA7 expression in breast cancer in validated cohort and TCGA cohort.

Notes: (A) ITGA7 expression was examined by RT-qPCR in 36 paired human breast cancer tissues and adjacent noncancerous tissues (paired $t$-test, $P<0.00 \mathrm{I}$ ). A logarithmic scale of $2^{-\Delta \Delta C t}$ is used to represent the fold change in quantitative real-time PCR detection. (B) The TCGA cohort contained I, I00 breast tumor tissues and II 3 normal tissues. RPKM is used to represent expression of ITGA7. The analysis was done using the Mann-Whitney $U$-test. $* * * P<0.001$ : $* * * * P<0.0001$.

Abbreviations: ITGA7, integrin subunit alpha 7; RPKM, reads per kilobases per million reads; RT-qPCR, real-time quantitative PCR; TCGA, The Cancer Genome Atlas. 
group according to the median value. The results revealed that lymph node metastasis $(P=0.030)$ and tumor size $(P=0.024)$ were significantly related to the $I T G A 7$ expression (Table 1). In the validated cohort, we divided all patients into the low-expression group $(n=18)$ and high-expression group $(n=18)$ according to the median value as same. The results showed that age $(P=0.169)$ and lymph node metastasis $(P=0.075)$ were not related to the expression of ITGA7 negatively (Table 2). These results indicated that low ITGA7 expression may influence the ability of migration of breast cancer cells and was associated with unfavorable prognosis in breast cancer.

\section{ITGA7 regulates migration and invasion of breast cancer lines}

To confirm the role of ITGA7 in breast cancer, we assessed ITGA7 expression level in several breast cancer cell lines and normal breast cell lines by using RT-qPCR. We found that expression level of ITGA7 was higher in MDA-MB-231 and BT-549 than in other breast cell lines (Figure 2A). So, we selected MDA-MB-231 and BT-549 for further experiments. To further examine whether ITGA7 functions in breast cancer progression, we knocked down ITGA7 expression in MDA-MB-231 and BT-549 using siRNA. As can be seen in

Table I The relationship between ITGA7 and clinicopathologic characteristics in TCGA cohort

\begin{tabular}{|c|c|c|c|c|}
\hline $\begin{array}{l}\text { Clinicopathologic } \\
\text { characteristics }\end{array}$ & $\begin{array}{l}\text { Low } \\
\text { expression } \\
\text { (\%) }\end{array}$ & $\begin{array}{l}\text { High } \\
\text { expression } \\
\text { (\%) }\end{array}$ & $\chi^{2}$ & $P$-value \\
\hline Age (years) & & & 0.001 & 0.973 \\
\hline$\leq 60$ & 296 & 296 & & \\
\hline$>60$ & 243 & 242 & & \\
\hline Tumor size & & & 7.475 & $0.024 *$ \\
\hline$<2 \mathrm{~cm}$ & 123 & 155 & & \\
\hline $2-5 \mathrm{~cm}$ & 335 & 291 & & \\
\hline$>5 \mathrm{~cm}$ & 81 & 92 & & \\
\hline Lymph node metastasis & & & 8.951 & $0.030 *$ \\
\hline No & 256 & 274 & & \\
\hline NI & 195 & 160 & & \\
\hline N2 & 60 & 57 & & \\
\hline N3 & 28 & 47 & & \\
\hline Distant metastasis & & & 0.854 & 0.356 \\
\hline No & 530 & 427 & & \\
\hline Yes & 9 & 11 & & \\
\hline Clinical stage & & & 1.662 & 0.197 \\
\hline I-II & 415 & 396 & & \\
\hline III-IV & 124 & 142 & & \\
\hline
\end{tabular}

Note: $*$-value $<0.05$.

Abbreviations: ITGA7, integrin subunit alpha 7; TCGA, The Cancer Genome Atlas.
Table 2 The relationship between ITGA7 and clinicopathologic characteristics in the validated cohort

\begin{tabular}{|c|c|c|c|c|}
\hline $\begin{array}{l}\text { Clinicopathologic } \\
\text { characteristics }\end{array}$ & $\begin{array}{l}\text { Low } \\
\text { expression } \\
\text { (\%) }\end{array}$ & $\begin{array}{l}\text { High } \\
\text { expression } \\
\text { (\%) }\end{array}$ & $\chi^{2}$ & $P$-value \\
\hline Age & & & 1.893 & 0.169 \\
\hline$\leq 60$ & 17 & 14 & & \\
\hline$>60$ & 1 & 4 & & \\
\hline Tumor size & & & 20.029 & 0.598 \\
\hline$<2 \mathrm{~cm}$ & 10 & 9 & & \\
\hline $2-5 \mathrm{~cm}$ & 8 & 8 & & \\
\hline$>5 \mathrm{~cm}$ & 0 & 1 & & \\
\hline Lymph node metastasis & & & 3.167 & $0.075^{*}$ \\
\hline No & 6 & 10 & & \\
\hline Yes & 12 & 8 & & \\
\hline Distant metastasis & & & 0.001 & 0.97 \\
\hline No & 17 & 16 & & \\
\hline Yes & 1 & 2 & & \\
\hline Estrogen receptor & & & 0.106 & 0.744 \\
\hline Negative & 8 & 10 & & \\
\hline Positive & 10 & 8 & & \\
\hline Progesterone receptor & & & 0.106 & 0.744 \\
\hline Negative & 8 & 10 & & \\
\hline Positive & 10 & 8 & & \\
\hline Her-2 receptor & & & 0.432 & 0.511 \\
\hline Negative & 7 & 8 & & \\
\hline Positive & 11 & 10 & & \\
\hline Clinical stage & & & 4.886 & 0.067 \\
\hline I-II & 10 & 13 & & \\
\hline III-IV & 8 & 5 & & \\
\hline
\end{tabular}

Note: $* P$-value $<0.05$

Abbreviation: ITGA7, integrin subunit alpha 7.

Figure 2B-D, both mRNA and protein levels of ITGA7 were significantly reduced.

Previous studies have proven that a cancer cell's abilities of migration and invasion were correlated with tumor progression. ${ }^{15,16}$ We next examined whether knocking down ITGA7 affected the functions of breast cancer cell lines. Our results showed that downregulated ITGA7 significantly enhanced migration capacity of MDA-MB-231 and BT-549 compared with the control groups (Figure $3 \mathrm{~A}$ and $\mathrm{B}$ ). The invasion assays also showed that downregulated ITGA7 effectively enhanced invasion capacity of MDA-MB-231 and BT-549 (Figure 4A and B).

\section{ITGA7 regulates migration and invasion via addition to c-met-regulated vimentin}

We found that knockdown of ITGA7 led to significantly increased c-met in MDA-MB-231 and BT-549 (Figure 5A). In a previous study, ${ }^{10,28}$ repression of the c-Met/Akt/mTOR pathway was found to suppress EMT. Vimentin is important in EMT and tumor progression. ${ }^{17,18}$ In order to explore the 
A

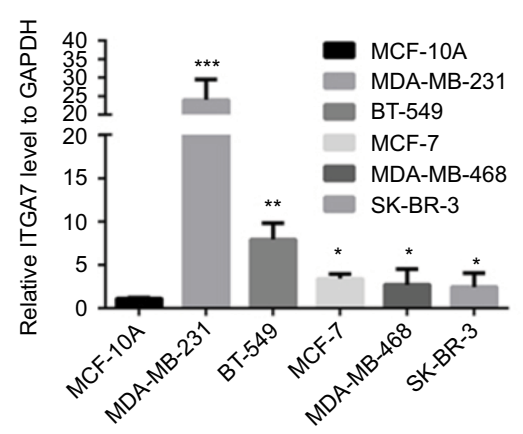

C

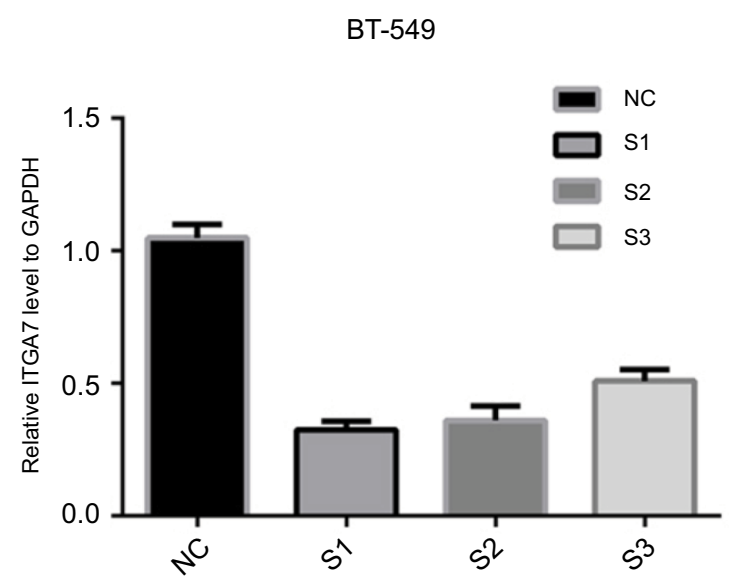

B

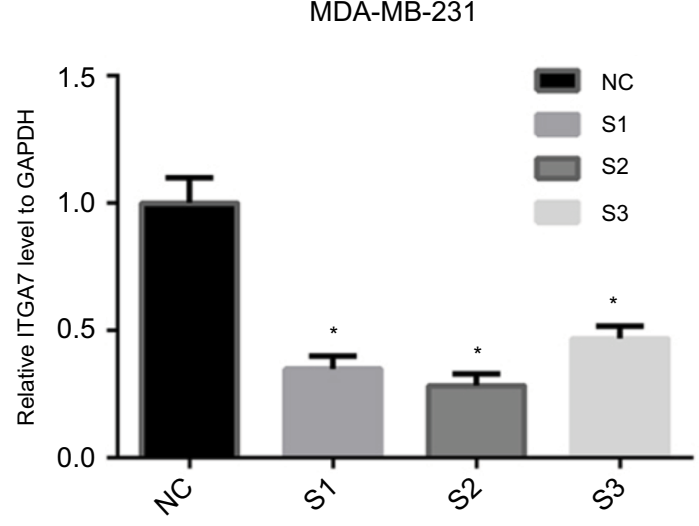

MDA-MB-231

D

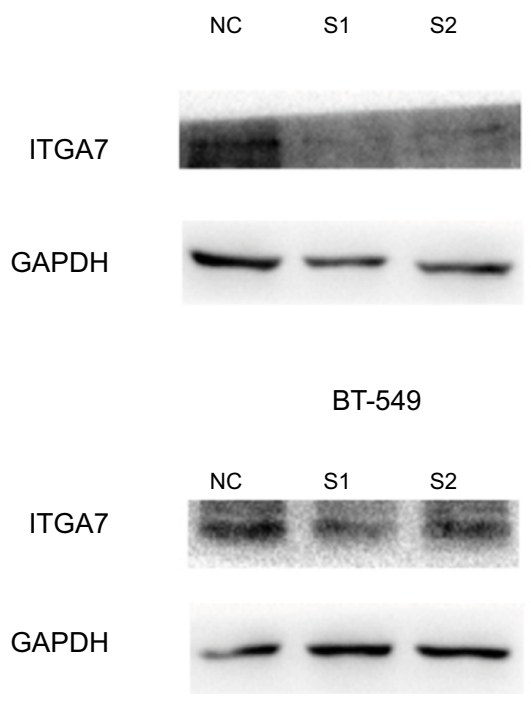

Figure 2 The expression level of ITGA7 in breast cancer cell lines.

Notes: (A) The relative expression of ITGA7 gene (compared with the GAPDH gene) using RT-qPCR. Compared to the other cell lines, ITGA7 was expressed at a higher level in MDA-MB-23I and BT-549. (B-D) MDA-MB-23I and BT-549 cells were transfected with siRNA-I, siRNA-2, or si-NC for 48 h, and both mRNA and protein levels of ITGA7 were significantly reduced. Compared with corresponding control group, the expression of ITGA7 gene in SI and S2 group was lower. We defined ITGA7 siRNA-I as SI, ITGA7 siRNA-2 as S2, and ITGA7 siRNA-3 as S3. ${ }^{*} P<0.05 ; * * P<0.01 ; * * * P<0.00$ I

Abbreviations: ITGA7, integrin subunit alpha 7; NC, negative control; RT-qPCR, real-time quantitative PCR.

potential mechanism of ITGA7 in breast cancer, we measured the expression level of epithelial markers and mesenchymal markers in MDA-MB-231 and BT-549 using Western blotting. The result showed that cells with knocked down ITGA7 had significantly higher vimentin expression level (Figure 5B). Our result showed that knockdown of ITGA7 may regulate vimentin via enhancing c-met.

\section{Discussion}

Breast cancer represents the highest health burden in the world, which is consistent with recently published data which reports that breast cancer is a significant part of the cause of cancer-related death in women worldwide. ${ }^{2}$
Although much progress in medical research had been made in breast cancer, ${ }^{19-21}$ there is still much left unknown about the molecular mechanisms of breast cancer. Some studies have demonstrated that ITGA7 gene was associated with malignant melanoma, prostate and liver carcinomas, and glioblastoma. ${ }^{12}$ However, there is still known little about its function in breast cancer.

In our study, the main aim was to prove the potential role of ITGA7 in breast cancer. We found that ITGA7 in breast cancer tissues was downregulated compared to paired adjacent nontumor tissues by RT-qPCR. This result was further identified in TCGA cohort. We analyzed the clinical features of breast cancer patients from TCGA and found that lymph 

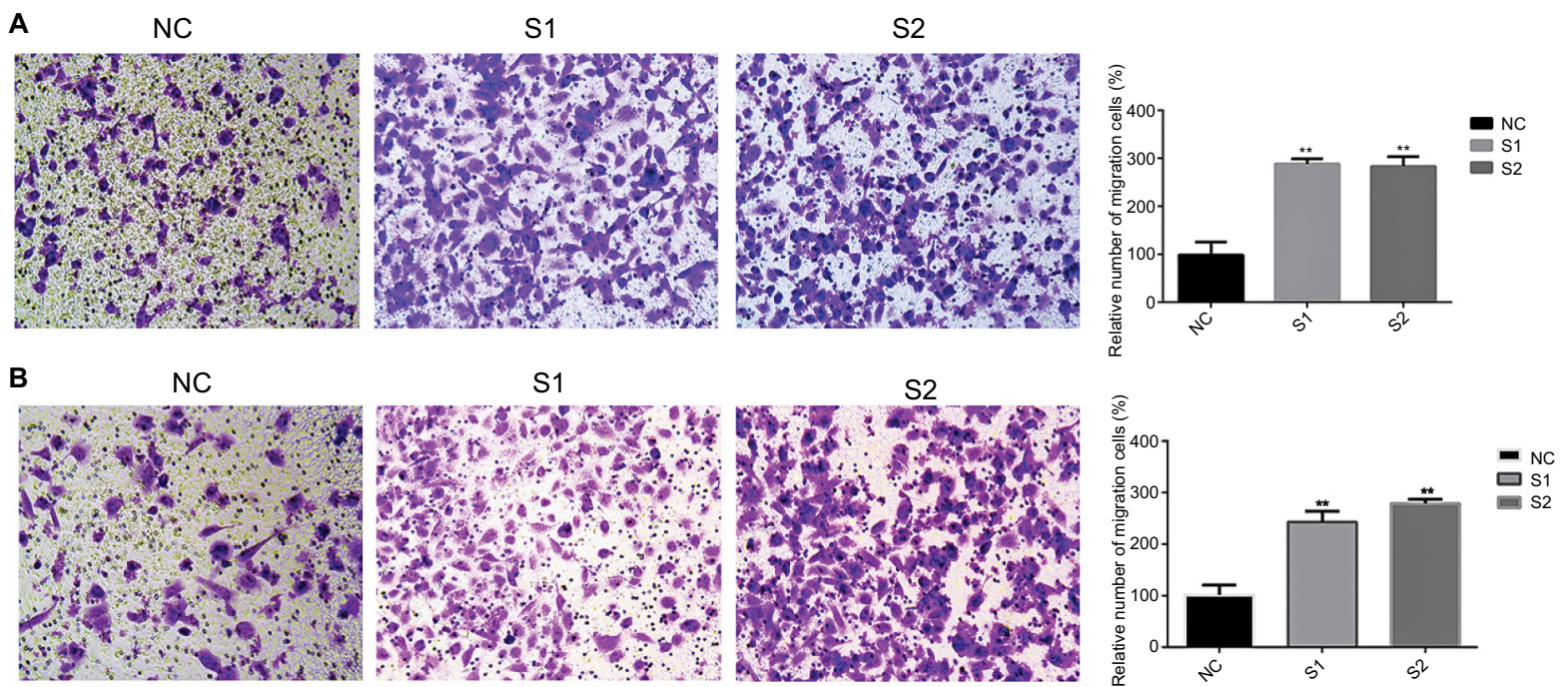

Figure 3 Downregulation of ITGA7 gene expression in MDA-MB-23I and BT-549 cells inhibits migration.

Notes: These experiments were done at least 3 independent times. (A) In MDA-MB-23I, Transwell migration assays in downregulation ITGA7 cells and their corresponding control cells. (B) In BT-549, Transwell migration assays in downregulation of ITGA7 cells and their corresponding control cells. Quantitative results of migration assays. The stained cells were then counted manually from 5 randomly selected fields and normalized with cell proliferation. ${ }^{* * P}<0.01$ in comparison with the NC group using Student's t-test. We defined ITGA7 siRNA-I as SI and ITGA7 siRNA-2 as S2.

Abbreviations: ITGA7, integrin subunit alpha 7; NC, negative control.

A

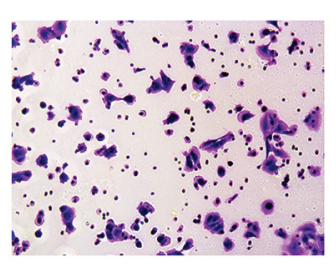

B

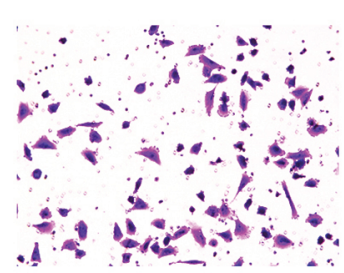

S1

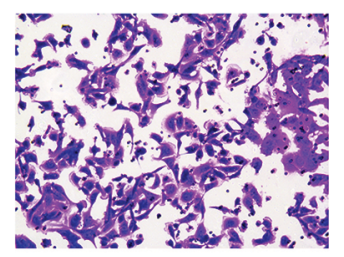

S1

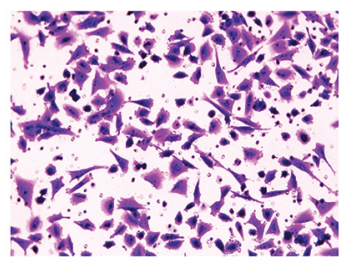

S2
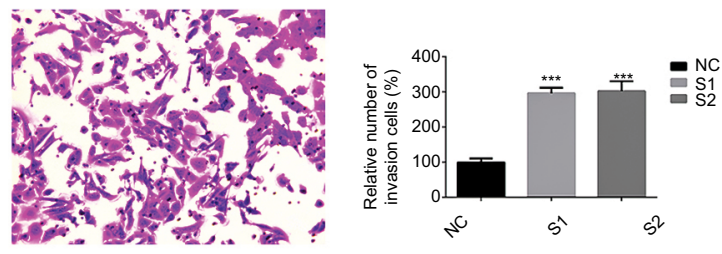

S2

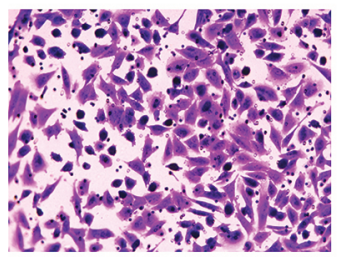

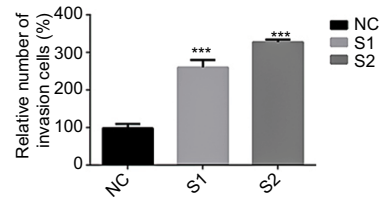

Figure 4 Downregulation of ITGA7 gene expression in MDA-MB-23I and BT-549 cells inhibits invasion.

Notes: These experiments were done at least 3 independent times. (A) Transwell invasion assays in downregulation ITGA7 cells and their corresponding NC cells in MDAMB-23I. (B) Transwell invasion assays in downregulation ITGA7 cells and their corresponding negative control cells in BT-549. Quantitative results of invasion assays. The stained cells were manually counted from 5 randomly selected fields and normalized with cell proliferation. ***P $<0.00 \mathrm{I}$ in comparison with the NC group using Student's t-test. We defined ITGA7 siRNA-I as SI and ITGA7 siRNA-2 as S2.

Abbreviations: ITGA7, integrin subunit alpha 7; NC, negative control.

node metastasis $(P=0.030)$ and tumor size $(P=0.024)$ were significantly related with the ITGA7 expression. These findings suggest that $I T G A 7$ may play an important role in breast cancer and encouraged us to proceed with the next step to study the $I T G A 7$ gene in cell lines. We found that ITGA7 was expressed at a higher level in MDA-MB-231 and BT-549 than in other breast cell lines. Then, using cellular and molecular technology, we found that knockdown of ITGA7 led to an increase in migration and invasion abilities, which is consistent with ITGA7 being associated with breast cancer.

In recent decades, the development of c-met has been increasingly recognized to play pivotal roles in promoting tumor process. ${ }^{22-24} \mathrm{Jia}$ et $\mathrm{al}^{25}$ found that inhibiting c-MET could enhance the response of the colorectal cancer cells 
A

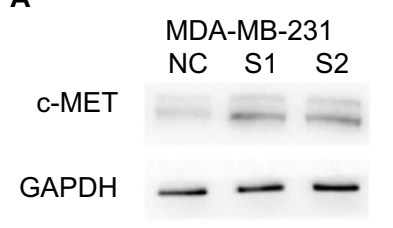

B

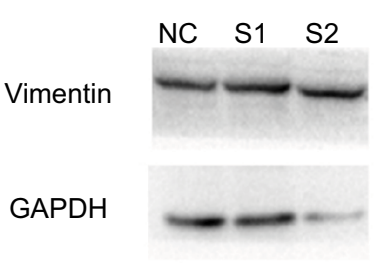

BT-549

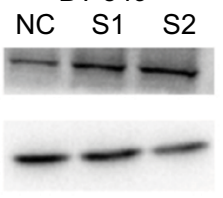

BT-549

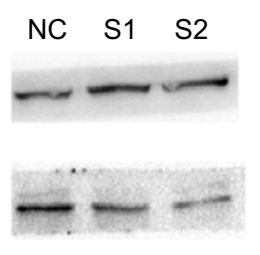

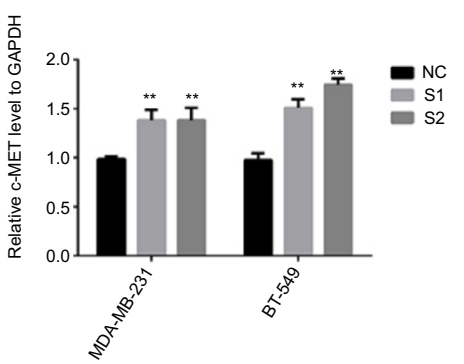

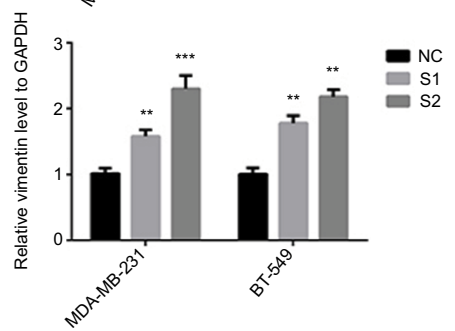

Figure 5 Downregulation of ITGA7 regulates migration and invasion via addition to c-met-regulated vimentin.

Notes: These experiments were done at least 3 independent times. We defined ITGA7 siRNA-I as SI and ITGA7 siRNA-2 as S2. (A) The influence of ITGA7 expression on c-MET in MDA-MB-23I and BT-549 cells by Western blot. (B) The influence of ITGA7 expression on vimentin in MDA-MB-23I and BT-549 cells by Western blot. $* * P<0.01$ and $* * * P<0.00 I$ in comparison with the NC group using Student's t-test. We defined ITGA7 siRNA-I as SI and ITGA7 siRNA-2 as S2.

Abbreviations: ITGA7, integrin subunit alpha 7; NC, negative control.

to irradiation in vitro and in vivo. Our study proved that knocking down ITGA7 could enhance c-met. EMT is a developmental multistep molecular process which may induce tumor process. ${ }^{26,27}$ Recently, EMT was found that facilitate the progression of breast cancer. It has been reported that repression of the c-Met/Akt/mTOR pathway could suppress EMT in breast cancer. ${ }^{10}$ So, we tested the EMT-related epithelial marker and the mesenchymal markers after ITGA7 knockdown. We found that the mesenchymal marker vimentin was induced after ITGA 7 knockdown. Together, these results mean that knockdown of ITGA7 enhances breast cancer cell migration and invasion via c-met regulated vimentin.

However, our study still has several limitations. For one thing, gain-of-function experiments need to be conducted to further validate the results. For another, we need to expand the validation group to provide reliable clinicopathological features and findings.

\section{Conclusion}

By using bioinformatics analysis and cellular and molecular approaches, the role of ITGA7 gene as a suppressor gene in breast cancer was demonstrated. We found that knockdown of ITGA 7 could enhance breast cancer cell migration and invasion. This study indicated that ITGA7 may act as a potential drug target in breast cancer.

\section{Acknowledgments}

This study was funded by Natural Science Foundation of Zhejiang Province (LY18H160053, LY17H160053, and
LY18H160053) and the Science and Technology Project of Wenzhou (Y20170030). The authors thank Yizuo Chen and Zhiqiang Ye for assistance help with data analysis.

\section{Disclosure}

The authors report no conflicts of interest in this work.

\section{References}

1. Jemal A, Bray F, Center MM, Ferlay J, Ward E, Forman D. Global cancer statistics. CA Cancer J Clin. 2011;61(2):69-90.

2. Siegel RL, Miller KD, Jemal A. Cancer statistics, 2017. CA Cancer J Clin. 2017;67(1):7-30.

3. Ahmed MI, Harvey JR, Kirby J, Ali S, Lennard TWJ. O-98 role of the chemokine receptor CXCR4 in breast cancer metastasis. Eur J Cancer Suppl. 2007;5(3):30.

4. van Zijl F, Krupitza G, Mikulits W. Initial steps of metastasis: cell invasion and endothelial transmigration. Mutat Res. 2011;728(1-2):23-34.

5. Pelkonen M, Luostari K, Tengström M, et al. Low expression levels of hepsin and TMPRSS3 are associated with poor breast cancer survival. BMC Cancer. 2015;15(1):431.

6. Zhao X, Qu J, Hui Y, et al. Clinicopathological and prognostic significance of c-Met overexpression in breast cancer. Oncotarget. 2017;8(34):56758-56767.

7. Gisterek I, Lata E, Halon A, et al. Prognostic role of c-met expression in breast cancer patients. Rep Pract Oncol Radiother. 2011;16(5):173-177.

8. Acloque H, Adams MS, Fishwick K, Bronner-Fraser M, Nieto MA. Epithelial-mesenchymal transitions: the importance of changing cell state in development and disease. J Clin Invest. 2009;119(6):1438-1449.

9. Tiwari N, Gheldof A, Tatari M, Christofori G. EMT as the ultimate survival mechanism of cancer cells. Semin Cancer Biol. 2012;22(3):194-207.

10. Hung CM, Kuo DH, Chou CH, Su YC, Ho CT, Way TD. Osthole suppresses hepatocyte growth factor (HGF)-induced epithelial-mesenchymal transition via repression of the c-Met/Akt/mTOR pathway in human breast cancer cells. J Agric Food Chem. 2011;59(17):9683-9690.

11. Song WK, Wang W, Foster R, Bielser DA, Kaufman SJ. H36-a7 is a novel integrin $\alpha$ chain that is developmentally regulated during skeletal myogenesis. J Cell Biol. 1992;117(3):643-657. 
12. Ziober BL, Chen YQ, Ramos DM, Waleh N, Kramer RH. Expression of the $\alpha 7 \beta 1$ laminin receptor suppresses melanoma growth and metastatic potential. Cell Growth Differ. 1999;10(7):479-490.

13. Ren B, Yu YP, Tseng GC, et al. Analysis of integrin $\alpha 7$ mutations in prostate cancer, liver cancer, glioblastoma multiforme, and leiomyosarcoma. J Natl Cancer Inst. 2007;99(11):868-880.

14. Yao CC, Ziober BL, Squillace RM, Kramer RH. $\alpha 7$ integrin mediates cell adhesion and migration on specific laminin isoforms. J Biol Chem. 1996;271(41):25598-25603.

15. Liu Y, Su C, Shan Y, Yang S, Ma G. Targeting Notch1 inhibits invasion and angiogenesis of human breast cancer cells via inhibition nuclear factor- $\mathrm{KB}$ signaling. Am J Transl Res. 2016;8(6):2681-2692.

16. Lee H, Goodarzi H, Tavazoie SF, Alarcon CR. TMEM2 is a SOX4regulated gene that mediates metastatic migration and invasion in breast cancer. Cancer Res. 2016;76(17):4994-5005.

17. Brzozowa M, Wyrobiec G, Kolodziej I, et al. The aberrant overexpression of vimentin is linked to a more aggressive status in tumours of the gastrointestinal tract. Prz Gastroenterol. 2015;10(1):7-11.

18. Chen C, Zimmermann M, Tinhofer I, Kaufmann AM, Albers AE. Epithelial-to-mesenchymal transition and cancer stem(-like) cells in head and neck squamous cell carcinoma. Cancer Lett. 2013;338(1): 47-56.

19. Jung JH, Son SH, Kim DH, et al. CONSORT-independent prognostic value of asphericity of pretherapeutic F-18 FDG uptake by primary tumors in patients with breast cancer. Medicine (Baltimore). 2017;96(46): e8438.
20. Hromas R, Kim HS, Sidhu G, et al. The endonuclease EEPD1 mediates synthetic lethality in RAD52-depleted BRCA1 mutant breast cancer cells. Breast Cancer Res. 2017;19(1):122.

21. Qian K, Liu G, Tang Z, et al. The long non-coding RNA NEAT1 interacted with miR-101 modulates breast cancer growth by targeting EZH2. Arch Biochem Biophys. 2017;615:1-9.

22. Harun N, Costa P, Christophi C. Tumour growth stimulation following partial hepatectomy in mice is associated with increased upregulation of c-Met. Clin Exp Metastasis. 2014;31(1):1-14.

23. Harshman LC, Choueiri TK. Targeting the hepatocyte growth factor/c-Met signaling pathway in renal cell carcinoma. Cancer J. 2013;19(4):316-323.

24. Li Y, Wang J, Gao X, et al. c-Met targeting enhances the effect of irradiation and chemical agents against malignant colon cells harboring a KRAS mutation. PLoS One. 2014;9(11):e113186.

25. Jia Y, Dai G, Wang J, et al. c-MET inhibition enhances the response of the colorectal cancer cells to irradiation in vitro and in vivo. Oncol Lett. 2016;11(4):2879-2885.

26. Luo Z, Li Y, Zuo M, et al. Effect of NR5A2 inhibition on pancreatic cancer stem cell (CSC) properties and epithelial-mesenchymal transition (EMT) markers. Mol Carcinog. 2017;56(5):1438-1448.

27. $\mathrm{Hu} \mathrm{SH}$, Wang $\mathrm{CH}$, Huang ZJ, et al. miR-760 mediates chemoresistance through inhibition of epithelial mesenchymal transition in breast cancer cells. Eur Rev Med Pharmacol Sci. 2016;20(23):5002-5008.

28. Chen QY, Jiao DM, et al. MiR-206 inhibits HGF-induced epithelialmesenchymal transition and angiogenesis in non-small cell lung cancer via c-Met/PI3k/Akt/mTOR pathway. Oncotarget. 2016;7(14):18247-18261.
Cancer Management and Research

\section{Publish your work in this journal}

Cancer Management and Research is an international, peer-reviewed open access journal focusing on cancer research and the optimal use of preventative and integrated treatment interventions to achieve improved outcomes, enhanced survival and quality of life for the cancer patient. The manuscript management system is completely online and includes

\section{Dovepress}

a very quick and fair peer-review system, which is all easy to use. Visit http://www.dovepress.com/testimonials.php to read real quotes from published authors. 